\title{
New proofs on two recent inequalities for unitarily invariant norms
}

Junjian Yang ${ }^{1,2,3,4^{*}}$ and Linzhang Lu $u^{1,5}$

\section{"Correspondence:}

junjianyang1981@163.com

'School of Mathematical Sciences,

Guizhou Normal University,

Guiyang, P.R. China

${ }^{2}$ School of Mathematics and

Statistics, Hainan Normal University, Haikou, P.R. China

Full list of author information is available at the end of the article

\begin{abstract}
In this short note, we provide alternative proofs for several recent results due to Audenaert (Oper. Matrices 9:475-479, 2015) and Zou (J. Math. Inequal. 10:1119-1122, 2016; Linear Algebra Appl. 552:154-162, 2019).
\end{abstract}

MSC: $15 \mathrm{~A} 45 ; 15 \mathrm{~A} 60$

Keywords: Arithmetic-geometric mean inequality; Hölder inequality; Unitarily invariant norm; Norm inequality

\section{Introduction}

Let $\mathbb{M}_{n}$ be the set of $n \times n$ complex matrices. For $A \in \mathbb{M}_{n}$, the singular values and eigenvalues of $A$ are denoted by $\sigma_{i}(A)$ and $\lambda_{i}(A)$, respectively, $i=1, \ldots, n$. The singular values $\sigma_{1}(A), \sigma_{2}(A), \ldots, \sigma_{n}(A)$ of a matrix $A$ are the eigenvalues of $|A|=\left(A^{*} A\right)^{\frac{1}{2}}$ arranged in decreasing order and repeated according to multiplicity. The Ky Fan $k$-norm, a particular unitarily invariant norm, is defined as $\|\cdot\|_{(k)}=\sum_{j=1}^{k} \sigma_{j}(A), 1 \leq k \leq n$. If $A$ is Hermitian, then all eigenvalues of $A$ are real and ordered as $\lambda_{1}(A) \geq \cdots \geq \lambda_{n}(A)$.

Let $A, B \in \mathbb{M}_{n}$. Bhatia and Kittaneh [8] proved an arithmetic-geometric mean inequality for unitarily invariant norms

$$
\left\|A^{*} B\right\| \leq \frac{1}{2}\left\|A A^{*}+B B^{*}\right\| .
$$

As a generalization of (1), Bhatia and Davis [7] proved that

$$
\left\|A^{*} X B\right\| \leq \frac{1}{2}\left\|A A^{*} X+X B B^{*}\right\|
$$

for $A, X, B \in \mathbb{M}_{n}$.

Albadawi [3] obtained a stronger version of the Hölder inequality for unitarily invariant norms. Let $A, X, B \in \mathbb{M}_{n}$ and $\frac{1}{p}+\frac{1}{q}=1, p, q>1, r \geq 0$. Then

$$
\left\|\left|A^{*} X B\right|^{r}\right\| \leq\left\|\left|A A^{*} X\right|^{\frac{r p}{2}}\right\|^{\frac{1}{p}}\left\|\left|X B B^{*}\right|^{\frac{r q}{2}}\right\|^{\frac{1}{q}}
$$

(c) The Author(s) 2020. This article is licensed under a Creative Commons Attribution 4.0 International License, which permits use, sharing, adaptation, distribution and reproduction in any medium or format, as long as you give appropriate credit to the original author(s) and the source, provide a link to the Creative Commons licence, and indicate if changes were made. The images or other third party material in this article are included in the article's Creative Commons licence, unless indicated otherwise in a credit line to the material. If material is not included in the article's Creative Commons licence and your intended use is not permitted by statutory regulation or exceeds the permitted use, you will need to obtain permission directly from the copyright holder. To view a copy of this licence, visit http://creativecommons.org/licenses/by/4.0/. 
which is a generalization of Horn and Zhan's result [10] (also called the Hölder inequality)

$$
\left\|\left|A^{*} B\right|^{r}\right\| \leq\left\|\left(A A^{*}\right)^{\frac{r p}{2}}\right\|^{\frac{1}{p}}\left\|\left(B B^{*}\right)^{\frac{r q}{2}}\right\|^{\frac{1}{q}} .
$$

Recently, Audenaert [5] proved that if $A, B \in \mathbb{M}_{n}$ and $\frac{1}{p}+\frac{1}{q}=1, p, q>1, r \geq 0, \alpha \in[0,1]$, then

$$
\left\|\left|A^{*} B\right|^{r}\right\| \leq\left\|\left(\alpha A A^{*}+(1-\alpha) B B^{*}\right)^{r p / 2}\right\|^{\frac{1}{p}}\left\|\left((1-\alpha) A A^{*}+\alpha B B^{*}\right)^{r p / 2}\right\|^{\frac{1}{q}},
$$

which is a unification of inequalities (1) and (4). By setting $r=1$ and $p=p^{\prime}=2$ in (5) we have

$$
\left\|\left|A^{*} B\right|\right\| \leq\left\|\alpha A A^{*}+(1-\alpha) B B^{*}\right\|^{\frac{1}{2}}\left\|(1-\alpha) A A^{*}+\alpha B B^{*}\right\|^{\frac{1}{2}} .
$$

Lin [12] gave a new proof of inequality (6). Zou and Jiang [16] generalized it to the following inequality: Let $A, B, X \in \mathbb{M}_{n}$ and $q \in[0,1]$. Then

$$
\left\|A X B^{*}\right\|^{2} \leq\left\|q A^{*} A X+(1-q) X B^{*} B\right\|\left\|(1-q) A^{*} A X+q X B^{*} B\right\| .
$$

Al-khlyleh and Kittaneh [2, Theorem 2.5] presented an inequality that refines inequality (7) for the particular unitarily invariant norm, Hilbert-Schmidt norm. For more results on interpolation between the arithmetic-geometric mean inequality and the CauchySchwarz inequality for matrices, see [1].

In this paper, we provide alternative proofs of inequalities (5) and (7), which provide new perspectives to the elegant results.

\section{Main results}

For presenting the new proofs, we need the following several lemmas.

Lemma 2.1 (see [6, Proposition IX.1.2]) Let $A, B \in \mathbb{M}_{n}$ be any two matrices such that the product $A B$ is Hermitian. Then, for every unitarily invariant norm, we have

$$
\|A B\| \leq\|\mathfrak{R}(B A)\| .
$$

Lemma 2.2 (see [6, p. 41]) Let $A, B \in \mathbb{M}_{n}$ and suppose that $f$ is convex and increasing on $[0, \infty)$. If

$$
\sum_{j=1}^{k} \sigma_{j}(A) \leq \sum_{j=1}^{k} \sigma_{j}(B), \quad k=1, \ldots, n,
$$

then

$$
\sum_{j=1}^{k} f\left(\sigma_{j}(A)\right) \leq \sum_{j=1}^{k} f\left(\sigma_{j}(B)\right), \quad k=1, \ldots, n .
$$


Lemma 2.3 (see [6, p. 35]) Let $A, B \in \mathbb{M}_{n}$. Then

$$
\sum_{j=1}^{k} \sigma_{j}(A+B) \leq \sum_{j=1}^{k} \sigma_{j}(A)+\sigma_{j}(B), \quad k=1, \ldots, n .
$$

Lemma 2.4 (see $\left[14\right.$, p. 63]) If $A \in \mathbb{M}_{n}$, then

$$
\lambda_{j}(\Re A) \leq \sigma_{j}(A), \quad j=1, \ldots, n .
$$

Lemma 2.5 (see [4] and [13, p. 228]) Let $A, B \in \mathbb{M}_{n}$ be positive semidefinite and $0 \leq q \leq 1$.

Then

$$
\sigma_{j}\left(A^{q} B^{1-q}\right) \leq \sigma_{j}(q A+(1-q) B), \quad j=1, \ldots, n .
$$

Audenaert [5] proved the following theorem. We give a different proof of the result.

Theorem 2.6 Let $A, B \in \mathbb{M}_{n}$ and $\frac{1}{p}+\frac{1}{q}=1, p, q>1, r \geq 0, \alpha \in[0,1]$. Then

$$
\left\|\left|A^{*} B\right|^{r}\right\| \leq\left\|\left(\alpha A A^{*}+(1-\alpha) B B^{*}\right)^{\frac{r p}{2}}\right\|^{\frac{1}{p}}\left\|\left((1-\alpha) A A^{*}+\alpha B B^{*}\right)^{\frac{r q}{2}}\right\|^{\frac{1}{q}} .
$$

Proof By Fan's dominance theorem (see [11, Theorem 1.4]) (11) is equivalent to

$$
\left\|\left|A^{*} B\right|^{r}\right\|_{(k)} \leq\left\|\left(\alpha A A^{*}+(1-\alpha) B B^{*}\right)^{\frac{r p}{2}}\right\|_{(k)}^{\frac{1}{p}}\left\|\left((1-\alpha) A A^{*}+\alpha B B^{*}\right)^{\frac{r q}{2}}\right\|_{(k)}^{\frac{1}{q}}
$$

for $k=1, \ldots, n$.

First, let us show this inequality for the Ky Fan 1-norm, that is, the spectral norm:

$$
\begin{aligned}
\left\|\left|A^{*} B\right|^{r}\right\|_{(1)}^{2} & =\sigma_{1}^{2}\left(\left|A^{*} B\right|^{r}\right) \\
& =\lambda_{\max }\left(\left|A^{*} B\right|^{2 r}\right) \\
& =\lambda_{\max }^{r}\left(B B^{*} A A^{*}\right) \\
& =\lambda_{\max }^{r}\left[\left(B B^{*}\right)^{\alpha} A A^{*}\left(B B^{*}\right)^{1-\alpha}\right] \\
& \leq \sigma_{1}^{r}\left(\left(B B^{*}\right)^{\alpha} A A^{*}\left(B B^{*}\right)^{1-\alpha}\right) \\
& =\left\|\left(B B^{*}\right)^{\alpha} A A^{*}\left(B B^{*}\right)^{1-\alpha}\right\|_{(1)}^{r} \\
& \leq\left\|\left(B B^{*}\right)^{\alpha}\left(A A^{*}\right)^{1-\alpha}\right\|_{(1)}^{r}\left\|\left(A A^{*}\right)^{\alpha}\left(B B^{*}\right)^{1-\alpha}\right\|_{(1)}^{r},
\end{aligned}
$$

which means that

$$
\sigma_{1}^{2}\left(\left|A^{*} B\right|^{r}\right) \leq \sigma_{1}^{r}\left(\left(B B^{*}\right)^{\alpha}\left(A A^{*}\right)^{1-\alpha}\right) \sigma_{1}^{r}\left(\left(A A^{*}\right)^{\alpha}\left(B B^{*}\right)^{1-\alpha}\right) .
$$

Second, using a standard argument via the antisymmetric product (see [5, p. 18]), (13) yields

$$
\prod_{j=1}^{k} \sigma_{j}\left(\left|A^{*} B\right|^{r}\right) \leq \prod_{j=1}^{k} \sigma_{j}^{\frac{r}{2}}\left(\left(B B^{*}\right)^{\alpha}\left(A A^{*}\right)^{1-\alpha}\right) \prod_{j=1}^{k} \sigma_{j}^{\frac{r}{2}}\left(\left(A A^{*}\right)^{\alpha}\left(B B^{*}\right)^{1-\alpha}\right)
$$


for $k=1, \ldots, n$. Since weak log-majorization implies weak majorization (see, [9, p. 174]), by (10) we have

$$
\begin{aligned}
\sum_{j=1}^{k} \sigma_{j}\left(\left|A^{*} B\right|^{r}\right) & \leq \sum_{j=1}^{k} \sigma_{j}^{\frac{r}{2}}\left(\left(B B^{*}\right)^{\alpha}\left(A A^{*}\right)^{1-\alpha}\right) \sigma_{j}^{\frac{r}{2}}\left(\left(A A^{*}\right)^{\alpha}\left(B B^{*}\right)^{1-\alpha}\right) \\
& \leq \sum_{j=1}^{k} \sigma_{j}^{\frac{r}{2}}\left((1-\alpha) A A^{*}+\alpha B B^{*}\right) \sigma_{j}^{\frac{r}{2}}\left(\alpha A A^{*}+(1-\alpha) B B^{*}\right)
\end{aligned}
$$

for $k=1, \ldots, n$. The left-hand side is $\left\|\left|A^{*} B\right|^{r}\right\|_{(k)}$. By the Hölder inequality the right-hand side is bounded from above by

$$
\begin{gathered}
{\left[\sum_{j=1}^{k} \sigma_{j}^{\frac{r p}{2}}\left((1-\alpha) A A^{*}+\alpha B B^{*}\right)\right]^{\frac{1}{p}}\left[\sum_{j=1}^{k} \sigma_{j}^{\frac{r q}{2}}\left(\alpha A A^{*}+(1-\alpha) B B^{*}\right)\right]^{\frac{1}{q}}} \\
=\left\|\left((1-\alpha) A A^{*}+\alpha B B^{*}\right)^{\frac{r p}{2}}\right\|_{(k)}^{\frac{1}{p}}\left\|\left(\alpha A A^{*}+(1-\alpha) B B^{*}\right)^{\frac{r q}{2}}\right\|_{(k)}^{\frac{1}{q}} .
\end{gathered}
$$

Thus (12) holds, and so does the conclusion. This completes the proof.

In fact, by a similar technique used in the theorem, we may present a new proof of the following result due to Zou [10], which is a unified version of inequalities (2) and (3).

Theorem 2.7 Let $A, B, X \in \mathbb{M}_{n}$ and $\frac{1}{p}+\frac{1}{q}=1, p, q>1, r \geq \max \left\{\frac{1}{p}, \frac{1}{q}\right\}, \alpha \in[0,1]$. Then

$$
\begin{aligned}
\left\|\left|A^{*} X B\right|^{2 r}\right\| \leq & \left\|\left(\alpha A A^{*} X+(1-\alpha) X B B^{*}\right)^{r p}\right\|^{\frac{1}{p}} \\
& \times\left\|\left((1-\alpha) A A^{*} X+\alpha X B B^{*}\right)^{r q}\right\|^{\frac{1}{q}} .
\end{aligned}
$$

Proof There is a subtle difference between the proof of (14) and that of the previous theorem although most techniques are similar. For the readers' convenience, we present the proof simply.

By Fan's dominance theorem (14) is equivalent to

$$
\begin{aligned}
\left\|\left|A^{*} X B\right|^{2 r}\right\|_{(k)} \leq & \left\|\left(\alpha A A^{*} X+(1-\alpha) X B B^{*}\right)^{r p}\right\|_{(k)}^{\frac{1}{p}} \\
& \times\left\|\left((1-\alpha) A A^{*} X+\alpha X B B^{*}\right)^{r q}\right\|_{(k)}^{\frac{1}{q}}
\end{aligned}
$$

for all $k=1, \ldots, n$.

If $X$ is a positive semidefinite matrix, then for Ky Fan 1-norm, we have

$$
\begin{aligned}
\left\|\left|A^{*} X B\right|^{2 r}\right\|_{(1)} & =\sigma_{1}\left(\left|A^{*} X B\right|^{2 r}\right) \\
& =\lambda_{\max }\left(\left|A^{*} X B\right|^{2 r}\right) \\
& =\lambda_{\max }^{r}\left(B^{*} X^{\frac{1}{2}} X^{\frac{1}{2}} A A^{*} X^{\frac{1}{2}} X^{\frac{1}{2}} B\right) \\
& =\lambda_{\max }^{r}\left(X^{\frac{1}{2}} B B^{*} X^{\frac{1}{2}} X^{\frac{1}{2}} A A^{*} X^{\frac{1}{2}}\right) \\
& =\lambda_{\max }^{r}\left(\left(X^{\frac{1}{2}} B B^{*} X^{\frac{1}{2}}\right)^{\alpha} X^{\frac{1}{2}} A A^{*} X^{\frac{1}{2}}\left(X^{\frac{1}{2}} B B^{*} X^{\frac{1}{2}}\right)^{1-\alpha}\right)
\end{aligned}
$$




$$
\begin{aligned}
\leq & \sigma_{1}^{r}\left(\left(X^{\frac{1}{2}} B B^{*} X^{\frac{1}{2}}\right)^{\alpha} X^{\frac{1}{2}} A A^{*} X^{\frac{1}{2}}\left(X^{\frac{1}{2}} B B^{*} X^{\frac{1}{2}}\right)^{1-\alpha}\right) \\
= & \left\|\left(X^{\frac{1}{2}} B B^{*} X^{\frac{1}{2}}\right)^{\alpha} X^{\frac{1}{2}} A A^{*} X^{\frac{1}{2}}\left(X^{\frac{1}{2}} B B^{*} X^{\frac{1}{2}}\right)^{1-\alpha}\right\|_{(1)}^{r} \\
\leq & \left\|\left(X^{\frac{1}{2}} B B^{*} X^{\frac{1}{2}}\right)^{\alpha}\left(X^{\frac{1}{2}} A A^{*} X^{\frac{1}{2}}\right)^{1-\alpha}\right\|_{(1)}^{r} \\
& \times\left\|\left(X^{\frac{1}{2}} A A^{*} X^{\frac{1}{2}}\right)^{\alpha}\left(X^{\frac{1}{2}} B B^{*} X^{\frac{1}{2}}\right)^{1-\alpha}\right\|_{(1)}^{r},
\end{aligned}
$$

which means that

$$
\begin{aligned}
\sigma_{1}^{2}\left(\left|A^{*} X B\right|^{r}\right) \leq & \sigma_{1}^{r}\left(\left(X^{\frac{1}{2}} B B^{*} X^{\frac{1}{2}}\right)^{\alpha}\left(X^{\frac{1}{2}} A A^{*} X^{\frac{1}{2}}\right)^{1-\alpha}\right) \\
& \times \sigma_{1}^{r}\left(\left(X^{\frac{1}{2}} A A^{*} X^{\frac{1}{2}}\right)^{\alpha}\left(X^{\frac{1}{2}} B B^{*} X^{\frac{1}{2}}\right)^{1-\alpha}\right) .
\end{aligned}
$$

Using a standard argument via the antisymmetric product (see [5, p. 18]), (15) yields

$$
\begin{aligned}
\prod_{j=1}^{k} \sigma_{j}^{2}\left(\left|A^{*} X B\right|^{r}\right) \leq & \prod_{j=1}^{k} \sigma_{j}^{r}\left(\left(X^{\frac{1}{2}} B B^{*} X^{\frac{1}{2}}\right)^{\alpha}\left(X^{\frac{1}{2}} A A^{*} X^{\frac{1}{2}}\right)^{1-\alpha}\right) \\
& \times \prod_{j=1}^{k} \sigma_{j}^{r}\left(\left(X^{\frac{1}{2}} A A^{*} X^{\frac{1}{2}}\right)^{\alpha}\left(X^{\frac{1}{2}} B B^{*} X^{\frac{1}{2}}\right)^{1-\alpha}\right)
\end{aligned}
$$

for $k=1, \ldots, n$. Since weak log-majorization implies weak majorization (see, [9, p. 174]), we have

$$
\begin{aligned}
\sum_{j=1}^{k} \sigma_{j}^{2}\left(\left|A^{*} X B\right|^{r}\right) \leq & \sum_{j=1}^{k} \sigma_{j}^{r}\left(\left(X^{\frac{1}{2}} B B^{*} X^{\frac{1}{2}}\right)^{\alpha}\left(X^{\frac{1}{2}} A A^{*} X^{\frac{1}{2}}\right)^{1-\alpha}\right) \\
& \times \sigma_{j}^{r}\left(\left(X^{\frac{1}{2}} A A^{*} X^{\frac{1}{2}}\right)^{\alpha}\left(X^{\frac{1}{2}} B B^{*} X^{\frac{1}{2}}\right)^{1-\alpha}\right) \\
\leq & \sum_{j=1}^{k} \sigma_{j}^{r}\left((1-\alpha)\left(X^{\frac{1}{2}} A A^{*} X^{\frac{1}{2}}\right)+\alpha\left(X^{\frac{1}{2}} B B^{*} X^{\frac{1}{2}}\right)\right) \\
& \times \sigma_{j}^{r}\left(\alpha\left(X^{\frac{1}{2}} A A^{*} X^{\frac{1}{2}}\right)+(1-\alpha)\left(X^{\frac{1}{2}} B B^{*} X^{\frac{1}{2}}\right)\right)
\end{aligned}
$$

for $k=1, \ldots, n$. The left-hand side is $\left\|\left|A^{*} X B\right|^{2 r}\right\|_{(k)}$. By the Hölder inequality the right-hand side is bounded from above by

$$
\begin{aligned}
& {\left[\sum_{j=1}^{k} \sigma_{j}^{r p}\left((1-\alpha)\left(X^{\frac{1}{2}} A A^{*} X^{\frac{1}{2}}\right)+\alpha\left(X^{\frac{1}{2}} B B^{*} X^{\frac{1}{2}}\right)\right)\right]^{\frac{1}{p}}} \\
& \quad \times\left[\sum_{j=1}^{k} \sigma_{j}^{r q}\left(\alpha\left(X^{\frac{1}{2}} A A^{*} X^{\frac{1}{2}}\right)+(1-\alpha)\left(X^{\frac{1}{2}} B B^{*} X^{\frac{1}{2}}\right)\right)\right]^{\frac{1}{q}} \\
& =\left\|\left((1-\alpha) X^{\frac{1}{2}} A A^{*} X^{\frac{1}{2}}+\alpha X^{\frac{1}{2}} B B^{*} X^{\frac{1}{2}}\right)^{r p}\right\|_{(k)}^{\frac{1}{p}} \\
& \quad \times\left\|\left(\alpha X^{\frac{1}{2}} A A^{*} X^{\frac{1}{2}}+(1-\alpha) X^{\frac{1}{2}} B B^{*} X^{\frac{1}{2}}\right)^{r q}\right\|_{(k)}^{\frac{1}{q}} .
\end{aligned}
$$


Thus

$$
\begin{aligned}
\left\|\left|A^{*} X B\right|^{2 r}\right\|_{(k)} \leq & \left\|\left((1-\alpha) X^{\frac{1}{2}} A A^{*} X^{\frac{1}{2}}+\alpha X^{\frac{1}{2}} B B^{*} X^{\frac{1}{2}}\right)^{r p}\right\|_{(k)}^{\frac{1}{p}} \\
& \times\left\|\left(\alpha X^{\frac{1}{2}} A A^{*} X^{\frac{1}{2}}+(1-\alpha) X^{\frac{1}{2}} B B^{*} X^{\frac{1}{2}}\right)^{r q}\right\|_{(k)}^{\frac{1}{q}} .
\end{aligned}
$$

Since $(1-\alpha) X^{\frac{1}{2}} A A^{*} X^{\frac{1}{2}}+\alpha X^{\frac{1}{2}} B B^{*} X^{\frac{1}{2}}$ and $\alpha X^{\frac{1}{2}} A A^{*} X^{\frac{1}{2}}+(1-\alpha) X^{\frac{1}{2}} B B^{*} X^{\frac{1}{2}}$ are Hermitian, since $r \geq \max \left\{\frac{1}{p}, \frac{1}{q}\right\}$, the previous inequalities become

$$
\begin{aligned}
& \left\|\left|A^{*} X B\right|^{2 r}\right\|_{(k)} \\
& \leq\left\|\left(\frac{(1-\alpha) A A^{*} X+\alpha B B^{*} X+(1-\alpha) X A A^{*}+\alpha X B B^{*}}{2}\right)^{r p}\right\|_{(k)}^{\frac{1}{p}} \\
& \quad \times\left\|\left(\frac{\alpha A A^{*} X+(1-\alpha) B B^{*} X+\alpha X A A^{*}+\alpha X B B^{*}}{2}\right)^{r q}\right\|_{(k)}^{\frac{1}{q}}
\end{aligned}
$$

(by (8) and Lemma 2.2)

$$
\leq\left\|\left((1-\alpha) A A^{*} X+\alpha X B B^{*}\right)^{r p}\right\|_{(k)}^{\frac{1}{p}} \times\left\|\left(\alpha A A^{*} X+(1-\alpha) X B B^{*}\right)^{r q}\right\|_{(k)}^{\frac{1}{q}} .
$$

(by Lemmas 2.2 and 2.3)

Next, we consider the case where $X$ is any matrix. By the singular value decomposition we know that there exist unitary matrices $U$ and $V$ such that $X=U D V^{*}$, and then by (16) we have

$$
\begin{aligned}
\left\|\left|A^{*} X B\right|^{2 r}\right\|_{(k)}= & \left\|\left|A^{*} U D V^{*} B\right|^{2 r}\right\|_{(k)} \\
\leq & \|\left(\frac{(1-\alpha)\left(A^{*} U\right)^{*}\left(A^{*} U\right) D+\alpha\left(V^{*} B\right)\left(V^{*} B\right)^{*} D}{2}\right. \\
& \left.+\frac{(1-\alpha) D\left(A^{*} U\right)^{*}\left(A^{*} U\right)+\alpha D\left(V^{*} B\right)\left(V^{*} B\right)^{*}}{2}\right)^{r p} \|^{2} \\
& \times \|\left(\frac{\alpha\left(A^{*} U\right)^{*}\left(A^{*} U\right) D+(1-\alpha)\left(V^{*} B\right)\left(V^{*} B\right)^{*} D}{2}\right. \\
& \left.+\frac{\alpha D\left(A^{*} U\right)^{*}\left(A^{*} U\right)+(1-\alpha) D\left(V^{*} B\right)\left(V^{*} B\right)^{*}}{2}\right)^{r q} \|_{(k)}^{\frac{1}{q}} \\
\leq & \left\|\left((1-\alpha)\left(A^{*} U\right)^{*}\left(A^{*} U\right) D+\alpha D\left(V^{*} B\right)\left(V^{*} B\right)^{*}\right)^{r p}\right\|_{(k)}^{\frac{1}{p}} \\
& \times\left\|\left(\alpha\left(A^{*} U\right)^{*}\left(A^{*} U\right) D+(1-\alpha) D\left(V^{*} B\right)\left(V^{*} B\right)^{*}\right)^{r q}\right\|_{(k)}^{\frac{1}{q}} \\
\leq & \left\|\left[U^{*}\left((1-\alpha) A A^{*} X+\alpha X B B^{*}\right) V\right]^{r p}\right\|_{(k)}^{\frac{1}{p}} \\
& \times\left\|\left[U^{*}\left(\alpha A A^{*} X+(1-\alpha) X B B^{*}\right) V\right]^{r q}\right\|_{(k)}^{\frac{1}{q}} \\
= & \left\|\left((1-\alpha) A A^{*} X+\alpha X B B^{*}\right)^{r p}\right\|_{(k)}^{\frac{1}{p}} \\
& \times\left\|U^{*}\left(\alpha A A^{*} X+(1-\alpha) X B B^{*}\right)^{r q}\right\|_{(k)}^{\frac{1}{q}}, \\
(k) & \\
& \\
& \\
& \\
&
\end{aligned}
$$


where the last equality is due to the fact that $\left\|\left|U_{1}^{*} P U_{2}\right|^{r}\right\|=\left\||P|^{r}\right\|$ for any $P \in \mathbb{M}_{n}$ and unitary matrices $U_{1}, U_{2}$. This completes the proof.

Finally, we give an alterative proof of (7) due to Zou and Jiang [16, Theorem 2.1].

Theorem 2.8 Let $A, B, X \in \mathbb{M}_{n}$ and $q \in[0,1]$. Then

$$
\left\|A X B^{*}\right\|^{2} \leq\left\|q A^{*} A X+(1-q) X B^{*} B\right\| \times\left\|(1-q) A^{*} A X+q X B^{*} B\right\| .
$$

Proof First, consider the special case where $A, B, X$ are Hermitian and $A=B$. Then

$$
\begin{aligned}
\left\|A X B^{*}\right\| & =\left\|A X A^{*}\right\|=\|A X A\| \\
& \leq \frac{1}{2}\left\|A^{2} X+X A^{2}\right\| \quad(\text { by }(2)) \\
& =\left\|\Re\left(q A^{2} X+(1-q) X A^{2}\right)\right\| \\
& \leq\left\|q A^{2} X+(1-q) X A^{2}\right\| \quad(\text { by }(9)) \\
& =\left\|q A^{*} A X+(1-q) X B^{*} B\right\| .
\end{aligned}
$$

Similarly,

$$
\left\|A X B^{*}\right\| \leq\left\|(1-q) A^{*} A X+q X B^{*} B\right\| .
$$

Thus by (17) and (18)

$$
\left\|A X B^{*}\right\|^{2} \leq\left\|q A^{*} A X+(1-q) X B^{*} B\right\| \times\left\|(1-q) A^{*} A X+q X B^{*} B\right\|,
$$

which is just the desired inequality in this particular case.

Next, consider the more general situation where $A$ and $B$ are Hermitian and $X$ is any matrix. Let

$$
T=\left(\begin{array}{cc}
A & 0 \\
0 & B
\end{array}\right), \quad Y=\left(\begin{array}{cc}
0 & X \\
X^{*} & 0
\end{array}\right) .
$$

Then by the particular case considered before

$$
\begin{aligned}
\left\|T Y T^{*}\right\| & =\|T Y T\| \leq \frac{1}{2}\left\|T^{2} Y+Y T^{2}\right\| \\
& \leq\left\|q T^{2} Y+(1-q) Y T^{2}\right\| .
\end{aligned}
$$

Multiplying out the block-matrices, we have

$$
\begin{aligned}
& T Y T=\left(\begin{array}{cc}
0 & A X B \\
B X^{*} A & 0
\end{array}\right), \\
& \frac{1}{2} T^{2} Y+\frac{1}{2} Y T^{2}=\frac{1}{2}\left(\begin{array}{cc}
0 & A^{2} X+X B^{2} \\
B^{2} X^{*}+X^{*} A^{2} & 0
\end{array}\right) .
\end{aligned}
$$


Hence we obtain the following inequality from (19):

$$
\left\|\left(\begin{array}{cc}
0 & A X B \\
B X^{*} A & 0
\end{array}\right)\right\| \leq \frac{1}{2}\left\|\left(\begin{array}{cc}
0 & A^{2} X+X B^{2} \\
B^{2} X^{*}+X^{*} A^{2} & 0
\end{array}\right)\right\|,
$$

which means that

$$
\|A X B\| \leq \frac{1}{2}\left\|A^{2} X+X B^{2}\right\| .
$$

So by (17) we have

$$
\begin{aligned}
\left\|A X B^{*}\right\| & =\|A X B\| \\
& \leq \frac{1}{2}\left\|A^{2} X+X B^{2}\right\| \\
& =\left\|q A^{*} A X+(1-q) X B^{*} B\right\| .
\end{aligned}
$$

The following inequality can be proved in exactly the same way:

$$
\begin{aligned}
\left\|A X B^{*}\right\| & =\|A X B\| \\
& \leq\left\|(1-q) A^{2} X+q X B^{2}\right\| \\
& =\left\|(1-q) A^{*} A X+q X B^{*} B\right\| .
\end{aligned}
$$

In this case, from (20) and (21) we have

$$
\left\|A X B^{*}\right\|^{2} \leq\left\|q A^{*} A X+(1-q) X B^{*} B\right\| \times\left\|(1-q) A^{*} A X+q X B^{*} B\right\| .
$$

Finally, Let $A=U A_{1}$ and $B=V B_{1}$ be polar decompositions of $A$ and $B$. Then

$$
A^{*} A X+X B^{*} B=A_{1}^{*} U^{*} U A_{1} X+X B_{1}^{*} V^{*} V B_{1}=A_{1}^{2} X+X B_{1}^{2},
$$

whereas

$$
\left\|A X B^{*}\right\|=\left\|U A_{1} X B_{1}^{*} V^{*}\right\|=\left\|A_{1} X B_{1}^{*}\right\| .
$$

So the theorem follows from inequality (22). This completes the proof.

Acknowledgements

Not applicable.

\section{Funding}

The work is supported by Hainan Provincial Natural Science Foundation for High-level Talents grant no. 2019RC171, the Ministry of Education of Hainan grant no. Hnky2019ZD-13, China Scholarship Council grant no. 201908460006, the Ministry of Education of Hainan grant no. Hnky2019ZD-13, the Provincial Key Laboratory, Hainan Normal University grant no. JSKX201904, and National Natural Science Foundation of China grant 11671105.

Availability of data and materials Not applicable. 
Authors' contributions

Both authors contributed almost the same amount of work to the manuscript. Both authors read and approved the final manuscript.

\section{Author details}

${ }^{1}$ School of Mathematical Sciences, Guizhou Normal University, Guiyang, P.R. China. ${ }^{2}$ School of Mathematics and Statistics, Hainan Normal University, Haikou, P.R. China. ${ }^{3}$ Hainan Key Laboratory for Computational Science and Application, Haikou, P.R. China. ${ }^{4}$ Key Laboratory of Data Science and Intelligence Education of Hainan Normal University, Ministry of Education, Haikou, P.R. China. ${ }^{5}$ School of Mathematical Sciences, Xiamen University, Xiamen, P.R. China.

\section{Publisher's Note}

Springer Nature remains neutral with regard to jurisdictional claims in published maps and institutional affiliations.

Received: 9 January 2020 Accepted: 28 April 2020 Published online: 11 May 2020

\section{References}

1. Ahmad, A., Omar, H., Kittaneh, F.: Interpolating inequalities for functions of positive semidefinite matrices. Banach J. Math. Anal. 12, 955-969 (2018)

2. Al-khlyleh, M., Kittaneh, F.: Interpolating inequalities related to a recent result of Audenaert. Linear Multilinear Algebra 65, 922-929 (2017)

3. Albadawi, H.: Hölder-type inequalities involving unitarily invariant norms. Positivity 16, 255-270 (2012)

4. Ando, T.: Matrix Young inequalities. Oper. Theory, Adv. Appl. 75, 33-38 (1995)

5. Audenaert, K.M.R.: Interpolating between the arithmetic-geometric mean and Cauchy-Schwarz matrix norm inequalities. Oper. Matrices 9, 475-479 (2015)

6. Bhatia, R.: Matrix Analysis. Springer, New York (1997)

7. Bhatia, R., Davis, C.: More matrix forms of the arithmetic-geometric mean inequality. SIAM J. Matrix Anal. Appl. 14 132-136 (1993)

8. Bhatia, R., Kittaneh, F.: On the singular values of a product of operators. SIAM J. Matrix Anal. Appl. 11, 272-277 (1990)

9. Horn, R.A., Johnson, C.R.: Topics in Matrix Analysis. Cambridge University Press, New York (1991)

10. Horn, R.A., Zhan, X.: Inequalities for C-S seminorms and Lieb functions. Linear Algebra Appl. 291, 103-113 (1999)

11. Li, C.-K., Mathias, R.: Generalizations of Ky Fan's dominance theorem. SIAM J. Matrix Anal. Appl. 19, 89-106 (1998)

12. Lin, M.: Remarks on two recent results of Audenaert. Linear Algebra Appl. 489, 24-29 (2016)

13. Zhan, X.: Matrix Inequalities. Springer, Berlin (2002)

14. Zhan, X.: Matrix Theory. Am. Math. Soc., Providence (2013)

15. Zou, L.: Unification of the arithmetic-geometric mean and Hölder inequalities for unitarily invariant norms. Linear Algebra Appl. 552, 154-162 (2019)

16. Zou, L., Jiang, Y.: A note on interpolation between the arithmetic-geometric mean and Cauchy-Schwarz matrix norm inequalities. J. Math. Inequal. 10, 1119-1122 (2016)

\section{Submit your manuscript to a SpringerOpen ${ }^{\circ}$ journal and benefit from:}

- Convenient online submission

- Rigorous peer review

- Open access: articles freely available online

- High visibility within the field

- Retaining the copyright to your article

Submit your next manuscript at $\gg$ springeropen.com 